\title{
Eficácia de aditivo via tanque para combustível diesel na limpeza dos sistemas de pós-tratamento.
}

\author{
Eng $^{\circ}$ André Moura de Oliveira \\ Eng $^{\circ}$ Arley Barbosa da Silva \\ Promax Bardahl.
}

Eng $^{\circ}$ Marcus Edsel Vercelino Jr.

Lubrizol Aditivos do Brasil Ltda.

Dr. Charles C. Conconi

Mercedes-Benz do Brasil Ltda.

\begin{abstract}
Reduce the GHG is a global driver for all OEMs around the world looking for a more sustainable mobility. Regulations for exhaust emissions are increasingly restrictive and focused on reducing pollutants. To meet these requirements, aftertreatment system technologies are incorporated into diesel engines such as Selective Catalytic Reduction and Diesel Particulate Filter, associated with low-Sulphur fuel and low SAPS lubricants, to ensure these levels. To maintain the efficiency of these systems they must be free from obstruction. However, some applications do not allow the properly regeneration causing inefficiency. In this work we will demonstrate the results of a diesel additive, based on metallic ions, which allows the regeneration of the system without the need to repair or replace components and no damage to the engine lubricant. The study was based on a field test with 5 vehicles powered by diesel engines, Proconve L6 emissions limit, running 30 thousand $\mathrm{km}$ each. In the end of the test it was possible to prove the efficacy of the additive in the cleaning process, regardless of the technology used, whether SCR or DPF, thus avoiding component replacement, operational failures and non-compliance with the emissions regime in place.
\end{abstract}

\section{INTRODUÇÃO}

Conforme (SHADDICK; THOMAS; MUDU; RUGGERI et al., 2020), metade da população mundial está exposta ao aumento da poluição e, de acordo com a Organização Mundial da Saúde (OMS), cerca de 4,2 milhões de pessoas morrem por ano devido à presença de material particulado (MP) com tamanho menor do que 2,5 $\mu \mathrm{m}$ de diâmetro. Das fontes conhecidas de material particulado, o motor diesel é uma das fontes de emissão destas partículas.
Com isso, a comunidade europeia criou em 1988 a primeira norma sobre as emissões de poluentes em veículos, denominando Euro 0, que foi ao longo do tempo sendo mais restritiva com a criação de novas normas denominadas de Euro 1, 2, 3, 4 e agora no Brasil temos que atender à norma Proconve L6, que é similar aos limites da Euro 5. Essas normas possibilitaram a diminuição das emissões de material particulado de até $80 \%$ entre a Euro 0 e a Euro 5 .

Para possibilitar a redução da emissão, foram introduzidos sistemas de pós-tratamentos, sendo o DPF o mais utilizado para reduzir o MP - material particulado. Porém, este filtro precisa ser regenerado toda vez que apresentar limites próximos à saturação. Conforme (MENG; CHEN; LI; FANG et al., 2020), a sua não regeneração provoca o aumento de pressão no sistema, com a perda de potência do motor e, em casos extremos, a parada do veículo. A regeneração pode ocorrer de forma passiva, onde os gases de escape atingem temperaturas mínimas e possibilitam a reação de oxidação do material particulado, que basicamente corresponde a resíduo de carbono produzindo $\mathrm{CO}_{2}$ e água. Há também a regeneração ativa, onde ocorre a injeção secundária de combustível provocando a queima e a oxidação do material particulado retido no filtro, produzindo $\mathrm{CO}_{2}$ e água (BAI; TANG; WANG; LI, 2016). No entanto, em algumas aplicações onde não se consegue atingir a temperatura para a regeneração passiva ou ativa, o acúmulo de MP leva ao entupimento do DFP e a necessidade da parada do veículo para manutenção.

Vários estudos tentam solucionar esse problema com a adição de aditivos (COOK; RICHARDS, 2002) (CHOI; LIU; JEONG, 2009). Neste artigo iremos trabalhar com um aditivo à base de íons metálicos e que foi também estudado por (LACARRIERE; SEGUELONG; HARLE; FABRE, 
2015; LACARRIERE; SEGUELONG; SPIVEY; DAS, 2017; ROCHER; SEGUELONG; HARLE; LALLEMAND et al., 2011). Este aditivo pode ser colocado no tanque de combustível permitindo a limpeza do DPF. Considerando o processo de regeneração passiva, com pós injeção de combustível dentro da câmara de combustão durante o ciclo de exaustão, o aumento da diluição de combustível no lubrificante é esperado e este parâmetro deve ser controlado para evitar efeitos danoso ao motor e seus periféricos, como por exemplo o conjunto turbocompressor. Desta forma foi avaliado a diluição do lubrificante pelo combustível, o seu impacto nas características do óleo de motor durante o seu uso.

\section{INFORMAÇÕES DO TESTE}

O objetivo do teste foi avaliar a eficácia do aditivo de limpeza do sistema de pós-tratamento durante $30 \mathrm{mil}$ quilômetros com o combustível diesel brasileiro, ou seja, teor de biodiesel B10 e teor de enxofre S10, em condição real. Os veículos utilizados foram utilitários de carga da montadora Kia, modelo K-2.500, denominado Bongo. Foram cinco unidades monitoradas, com período programado para o teste de 30 mil quilômetros. A cada 10 mil quilômetros foi realizada manutenção preventiva nos veículos, seguindo a recomendação da montadora, para coleta das amostras e troca dos lubrificantes. Neste momento foi retirado o DPF, para avaliação visual e registro fotográfico da entrada e saída de gases. Utilizamos em três veículos o modelo A-B-A, ou seja, com aditivo-sem aditivo-com aditivo, até atingirem os 30 mil quilômetros rodados. A taxa de tratamento de aditivo inicialmente usada foi a de limpeza, inserindo $750 \mathrm{ml}$ do aditivo para cada 80 litros de diesel no primeiro abastecimento. Nos demais abastecimentos foi utilizada a taxa de manutenção, que é de $500 \mathrm{ml}$ para cada 80 litros de combustível.

PREMISSAS PARA A REALIZAÇÃO DO TESTE Inicialmente foram realizadas avaliações e medições nos veículos com ferramenta de diagnóstico (Scanner), sempre na partida a frio, com o veículo em marcha lenta.

Havendo qualquer ocorrência de não conformidade, era realizada a correção de falhas antes da liberação do veículo para serviço/operação.

Realizamos avaliação e coleta de dados semanal, com o devido registro.

Os veículos passaram por um plano de manutenção preventiva com troca de óleo e todos os filtros - antes de iniciar os testes.

Como boas práticas e para evitar erros, foram utilizados adesivos de advertência, alertando que o veículo estava em teste e para a necessidade do uso do aditivo ao reabastecer. Também foi realizada uma reunião para orientação dos colaboradores envolvidos, que diariamente usam o veículo para entrega de mercadorias aos clientes de varejo e empresas de consumo.

LUBRIFICANTES - Foram utilizados três tipos de lubrificantes durante $\mathrm{o}$ teste. Nas intervenções $\mathrm{e}$ manutenções foram realizadas análises completas dos lubrificantes.

\section{$\underline{\text { Seguem as Especificações: }}$}

1. Óleo de motor - SAE 5W-30 API SN-Plus (ACEA C3) - Lubrificante formulado para o teste.

2. Óleo de motor - SAE 5W-30 API SN (ACEA C3) Lubrificante original e recomendado pela montadora do veículo.

3. Óleo de motor - SAE 5W-30 API SN (ACEA C3) Produto disponível no mercado brasileiro.

Tabela 1: Dados dos veículos.

\begin{tabular}{|c|c|c|c|c|}
\hline IDENTIFICAÇÃO & KM INICIAL & KM FINAL & COMENTÁRIO & LUBRIFICANTE \\
\hline VÉ́́CULO N. ${ }^{\circ 1}$ & 72.608 & 102.293 & Referência; sem aditivo & Original \\
\hline VÉ́́cULO N.'2 & 92.599 & 122.737 & Com aditivo & Lubrificante de mercado \\
\hline VÉ́CULO N. ${ }^{\circ 3}$ & 128.524 & 158.981 & Com aditivo & Protótipo \\
\hline VEíCULO N. ${ }^{\circ} 4$ & 133.652 & 163.698 & Com aditivo & Original \\
\hline VEículo N. ${ }^{\circ 5}$ & 142.760 & 173.504 & Referência; sem aditivo & Protótipo \\
\hline
\end{tabular}

O ADITIVO UTILIZADO NO TESTE - O aditivo em questão foi adicionado no tanque de combustível junto com o diesel, sendo totalmente compatível, homogêneo e estável. Na sua composição possui íons metálicos que, ao participarem do processo de combustão, se misturam com os gases de escape, diminuindo a energia de ativação, funcionando como catalisador, proporcionando a regeneração passiva mesmo com a temperatura do sistema para regeneração passiva baixa, reduzindo desta forma a saturação do DPF.

O produto foi desenvolvido para auxiliar o processo de regeneração, onde em condições adversas, nos intensos e extensos ciclos de condução urbana, podem causar falhas no DPF, sendo as principais causas o aumento de fuligem e pressão no sistema, acendendo luz de advertência no painel, fazendo com que o veículo tenha a rotação limitada e, em casos mais graves, podendo levar à parada total do veículo. 
Tabela 2: Característica dos veículos.

\begin{tabular}{|c|c|}
\hline \multicolumn{2}{|c|}{ KIA BONGO MODELO K2500 } \\
\hline MOTOR & D4CB 2.5L Turbodiesel Intercooler \\
\hline Tіро & 4 cilíndros em linha \\
\hline Sistema de alimentação & Common-rail \\
\hline Cilindrada & 2.497 \\
\hline Comando & No cabeçote, DOHC, $16 \mathrm{~V}$ \\
\hline Taxa de compressão & $16,4: 1$ \\
\hline Potência máxima & $130,5 \mathrm{cv} / 3.800 \mathrm{rpm}$ \\
\hline Torque máximo & $26 \mathrm{Kgf.m} / 1.500 \mathrm{rpm}$ \\
\hline Volume de lubrificante com filtro (L) & 7.4 \\
\hline \multicolumn{2}{|l|}{ DIREÇÃO } \\
\hline Tipo & Hidráulica, pinhão e cremalheira \\
\hline Raio de giro & 4,93 \\
\hline \multicolumn{2}{|l|}{ TRANSMISSÃO } \\
\hline Manual & 6 marchas sincronizadas \\
\hline Tração & Traseira $4 \times 2$ \\
\hline \multicolumn{2}{|l|}{ FREIOS } \\
\hline Dianteiros & Discos ventilados \\
\hline \begin{tabular}{|l} 
Traseiros \\
\end{tabular} & Tambor \\
\hline \multicolumn{2}{|l|}{ PNEUS } \\
\hline Dianteiros & 195 R15 - 8PR \\
\hline Traseiros & $195 \mathrm{R} 15-8 \mathrm{PR}$ \\
\hline \multicolumn{2}{|l|}{ DIMENSÕES DO VEÍCULO (mm) } \\
\hline Comprimento & 4.825 \\
\hline Largura & 1.740 \\
\hline Altura & 1.995 \\
\hline Distância entre eixos & 2.415 \\
\hline Bitola (diant. / tras.) & $1.490 / 1.460$ \\
\hline Balanço traseiro & 1.205 \\
\hline \multicolumn{2}{|l|}{ PESOS (kg) } \\
\hline Em ordem de marcha & 1.862 \\
\hline Capacidade de carga (com carroceria) & 1.530 \\
\hline Capacidade de carga no chassi (sem carroceria) & 1.812 \\
\hline Peso bruto total & 3.392 \\
\hline P.B.T. por eixo (dianteiro / traseiro) & $1.352 / 2.040$ \\
\hline Capacidade máxima de tração & 3.892 \\
\hline \multicolumn{2}{|l|}{ OUTRAS ESPECIFICAÇÕES } \\
\hline Volume do tanque de combustivel (L) & 80 \\
\hline Número de ocupantes (incluindo o motorista) & 3 \\
\hline
\end{tabular}

\section{$\underline{\text { O Produto Possui os Seguintes Benefícios: }}$}

1. Intensificação do número de regenerações passiva no DPF.

2. Restauro rápido do desempenho do $\mathrm{DPF}$, reduzindo assim as emissões de poluentes e a fuligem.

3. Recuperação da potência do motor por meio da desobstrução do sistema de injeção e pós-tratamento, reduzindo a temperatura de combustão para uma regeneração ideal e completa do DPF.

4. Evita a incrustação e depósitos nos injetores de combustível, conforme estudado por (LACARRIERE; SEGUELONG; HARLE; FABRE, 2015-01-0896).

Modo de uso: para limpeza a taxa de tratamento é de $750 \mathrm{ml}$ para 80 litros de combustível e para manutenção a taxa de tratamento é de $500 \mathrm{ml}$ para 80 litros.

Aplicações: em todos os motores a diesel e com todos os tipos de sistema de pós-tratamento.
Particularmente indicado para condições de condução urbana severa e/ou quando o veículo opera em marcha lenta por longos períodos.

RESULTADO DO TESTE - Apresentamos neste trabalho o resultado do teste dos veículos que rodaram com e sem o uso do aditivo para limpeza do DPF.

Veículo sem Aditivo - Os veículos rodaram todo o teste, ou seja, 30 mil quilômetros sem o uso do aditivo no sistema. É possível observar na figura 1 um aumento da fuligem no DPF.

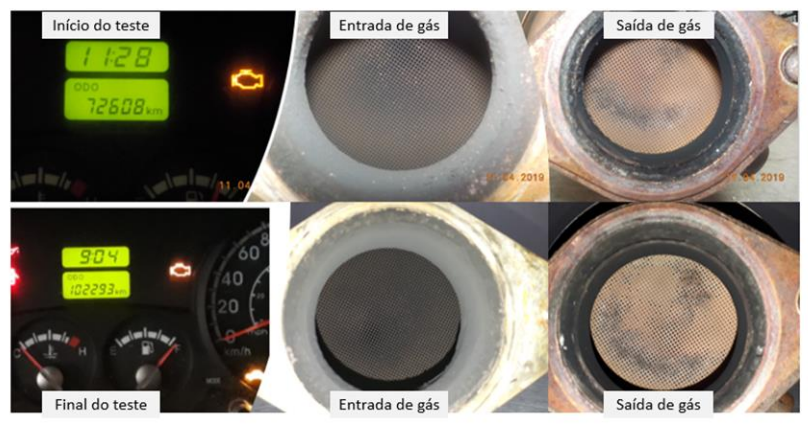

Figura 1: Veículo sem aditivo.

Veículo com Aditivo - Eles rodaram no modelo A-B$\mathrm{A}$, ou seja, os primeiros 10 mil quilômetros com o uso do aditivo. No período de 10 a 20 mil quilômetros, o veículo rodou sem o aditivo e, dos 20 aos 30 mil quilômetros, com o aditivo novamente. É possível observar visualmente uma redução do depósito de fuligem no DPF proporcionada pelo aumento de regeneração passiva do sistema em função do uso do produto.

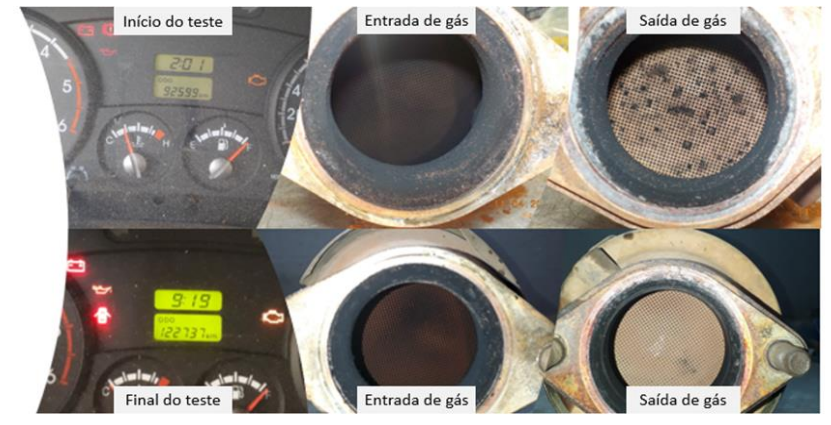

Figura 2: Veículo com aditivo.

RESULTADO DOS DADOS COLETADOS PELO SCANNER - Foi instalado uma ferramenta de diagnóstico no veículo, a ETAS ES592.1 da própria montadora, para avaliar a quantidade de regenerações num período de 6 mil quilômetros. Sendo que o veículo rodou 1,5 mil quilômetros sem aditivo e 4,5 mil quilômetros com o aditivo. 


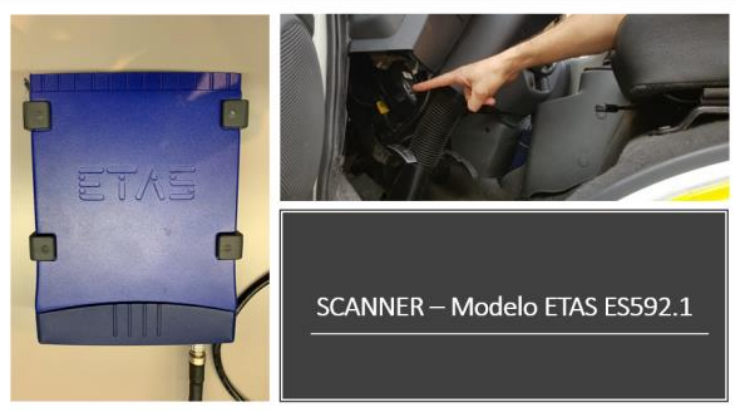

Figura 3: Scanner - Modelo ETAS ES592.1

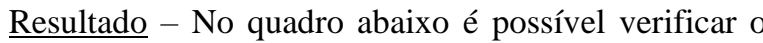
aumento da distância $[\mathrm{km}]$ entre regenerações ativas do veículo, operando na mesma rota, ciclo, condutor e combustível. A diferença se dá entre o fato de um período o diesel fora aditivado com o produto em questão, e no outro ele rodou sem o aditivo - diesel puro. É possível concluir com base no monitoramento via rede CAN que ao se operar com o aditivo no tanque, o número de regenerações passivas reduziu em média $67 \%$, conforme mostra a figura 4:

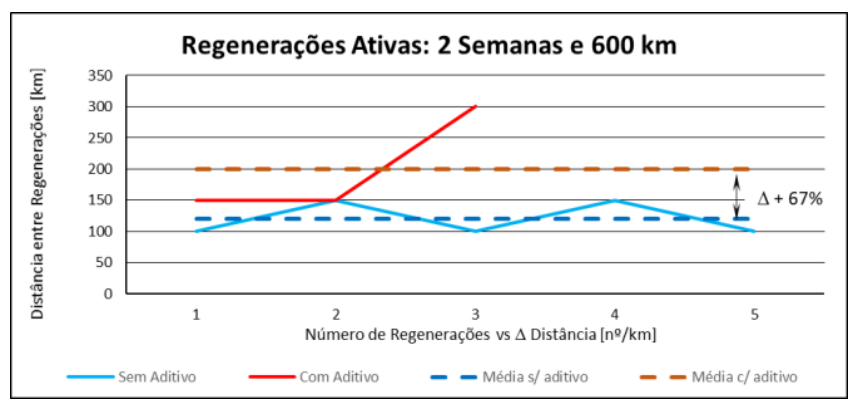

Figura 4: Regenerações ativas.

\section{RESULTADO}

DAS

ANÁLISES

DOS

LUBRIFICANTES - A primeira coleta foi no início do teste, depois com 5 mil, 10 mil, 20 mil e a última com 30 mil quilômetros rodados. As análises foram realizadas num laboratório independente e credenciado pelo INMETRO.

\section{As Principais Características Avaliadas Foram:}

1. Viscosidade - É definida como sendo a resistência que o fluido oferece ao escoamento. É uma das características mais importantes no controle do uso dos lubrificantes e deve ser mantida dentro dos limites estabelecidos para o serviço, que é de 9,3 a $12,5 \mathrm{~mm}^{2} / \mathrm{s}$ à $100^{\circ} \mathrm{C}$.

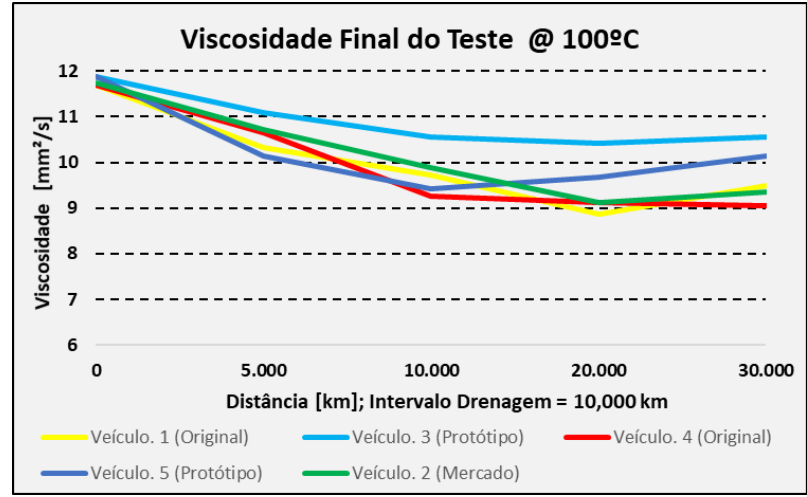

Figura 5: Viscosidade Cinemática a $100{ }^{\circ} \mathrm{C} ; \mathrm{mm}^{2} / \mathrm{s}$.

2. Fuligem - É o carbono resultado da reação incompleta do diesel. Embora a maior parte da fuligem produzida seja expelida pelos gases de escape, uma parte dela se mistura com o óleo do motor, que lubrifica os pistões e, consequentemente, é raspada pelos anéis em direção ao cárter. Uma parte da fuligem em suspensão nos gases também consegue passar pelas folgas normais dos anéis e se misturar facilmente com o óleo lubrificante e, por isto, é importante monitorá-la. Cada montadora especifica seus limites de fuligem que varia em função de cada projeto de motor. Os resultados apresentados na figura 6 demonstraram uma excelente capacidade de manter a fuligem em suspensão e dispersa no lubrificante.

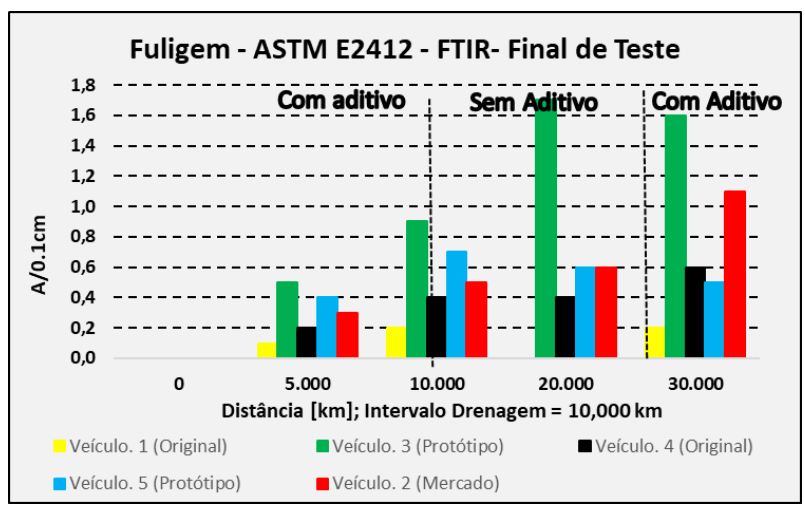

Figura 6: Fuligem ASTM E2412; A/0.1cm.

3. Diluição de Combustível - A análise de diluição por diesel utiliza a metodologia de ponto de fulgor (vaso fechado), indica se existe a contaminação por diesel, que por sua vez remete a um processo de combustão incompleta ou desfavorável. Quando este valor se aproxima de 5\%, recomenda-se análise mais aprofundada. 


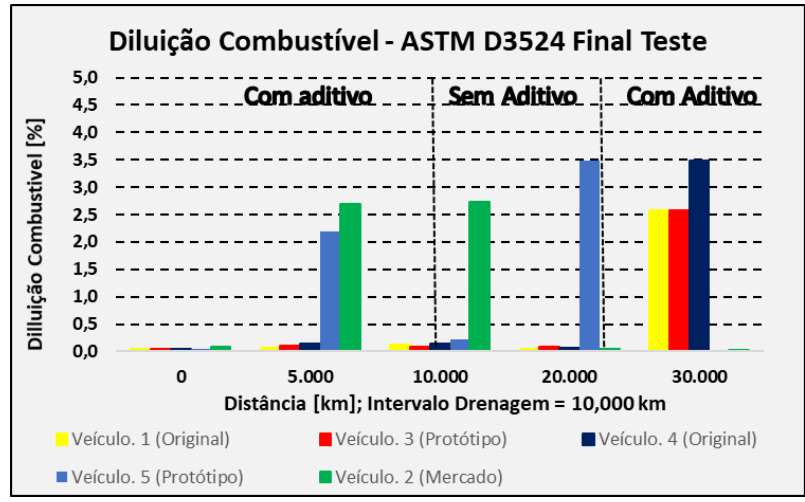

Figura 7: Diluição de combustível ASTM D3254; \%peso.

4. Oxidação - É a incorporação química do oxigênio e perda de desempenho do lubrificante devido ao seu tempo de uso, condições de operação adversas e anormais ou superaquecimento interno do motor. Quanto maior for o nível de oxidação, maior será o impacto na alteração da viscosidade do lubrificante.

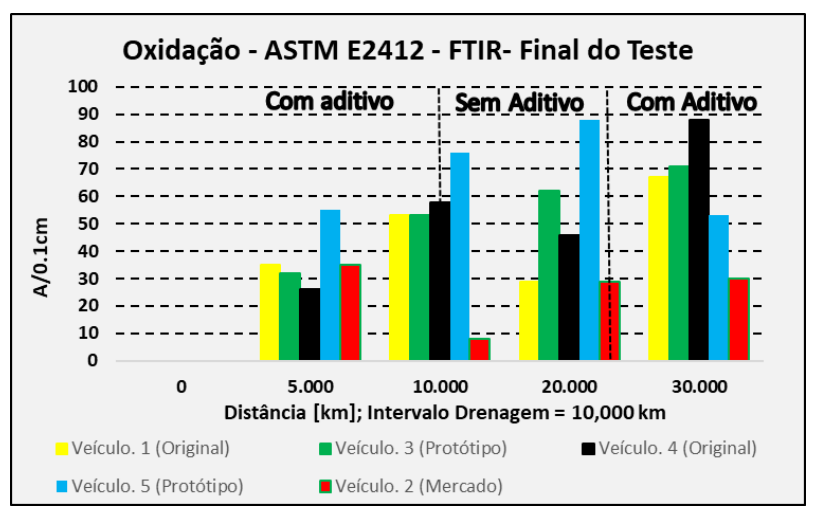

Figura 8: Oxidação ASTM E2412; A/0.1cm. observar:

Depois de rodar 30 mil $\mathrm{km}$ em campo, foi possível

1. Os três lubrificantes demonstraram um bom desempenho e não houve nenhum evento relacionado ao óleo durante o teste e tampouco a necessidade de reposição.

2. Independentemente da tecnologia de lubrificante utilizado no teste, foi observada oxidação nas amostras, mas não suficiente para alterar a viscosidade e nem comprometer o motor.

3. Uma diluição de combustível (entre 2 a 3,5\%) pode ser observada nas análises, em ambos os casos, com e sem aditivo.

4. A fuligem e a acidez foram controladas pelos aditivos presentes na tecnologia dos lubrificantes utilizados, sem afetar o motor e seu desempenho.
CONSUMO DE COMBUSTÍVEL - Ao avaliar o consumo de combustível, os veículos apresentaram irregularidades nos dados. Uma justificativa se deve ao fato de não utilizarem as mesmas rotas e nem os mesmos condutores durante o teste. Estes veículos são usados para realizar entregas nas cidades metropolitanas num raio de $100 \mathrm{~km}$ de Cajamar/SP. Em média os veículos trafegaram $70 \%$ em condições de tráfego urbano e $30 \%$ em condições de rodovia. A carga variou entre o peso máximo do veículo, ao sair para entrega, e sem carga no retorno para o local de origem do teste. $\mathrm{O}$ objetivo foi avaliar os veículos numa condição real de campo. Portanto, o uso do aditivo não teve impacto negativo no consumo de combustível.

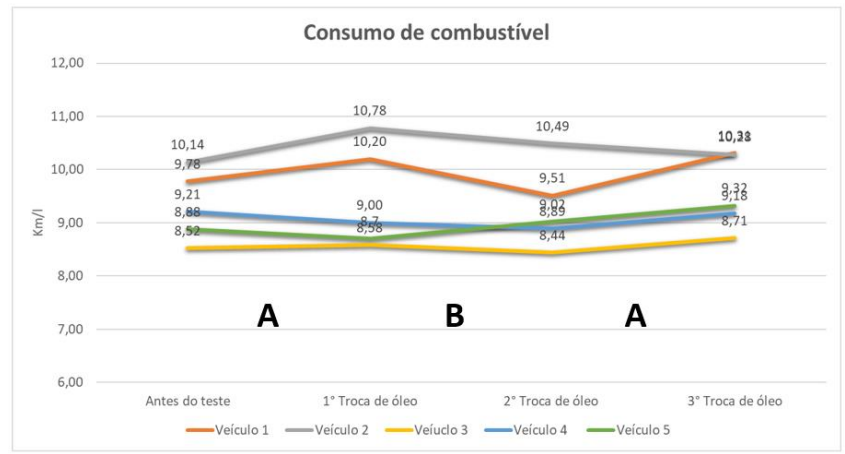

Figura 9: Consumo de combustível; km/l.

\section{CONCLUSÃO}

A tecnologia aplicada nos veículos equipados com DPF se mostrou eficaz na redução de depósitos de fuligem e não trouxe qualquer impacto negativo no equipamento, assim como no lubrificante utilizado no motor, conforme apresentado nos resultados das análises. Apesar de uma pequena diluição de combustível, não houve a presença elevada de ferro nas amostras, o que indica um desgaste normal do motor e o não comprometimento do lubrificante pelo uso do aditivo.

Os lubrificantes utilizados nos testes com características urbanas "anda e para" não sofreram alterações, mesmo trabalhando com um diesel contendo $10 \%$ de biodiesel.

O ciclo de operação dos veículos em teste demostrou que $70 \%$ do tempo de uso não possibilitaram a regeneração passiva do sistema e, nesse caso, o aditivo adicionado no tanque de combustível permitiu a redução da temperatura de ativação do processo em $150^{\circ} \mathrm{C}$, conforme estudado por (ROCHER; SEGUELONG; HARLE; LALLEMAND et al., 2011-01-0297). Nesta condição ocorreu a regeneração passiva do sistema, evitando com isso o uso da regeneração ativa, o qual pode trazer consequências negativas, como a possível diluição de combustível no óleo lubrificante. 
Nas futuras legislações de emissões no Brasil, Proconve P8 e L7, os DPFs serão utilizados para diminuir as emissões de MPs e esse aditivo se mostra uma opção de fácil e rápida aplicação quanto se trata de saturação por fuligem, sem a necessidade de substituição de componentes e veículo parado em um centro técnico.

\section{BIBLIOGRAFIA}

Rocher, L., Seguelong, T., Harle, V., Lallemand, M. Pudlarz, M. and Macduff, M., "New Generation Fuel Borne Catalyst for Reliable DPF Operation in Globally Diverse Fuels," SAE Technical Paper 2011-01-0297 (2011)

Lacarriere, A., Seguelong, T., Harle, V., and Fabre, C., "A Low Ash and Highly Stable Formulated Fuel Borne Catalyst with Injection System Deposit Prevention Properties," SAE Technical Paper 2015-01-0896 (2015)

Lacarriere, A., Seguelong, T., Spivey, D., and Das, A., "The Fuel-Borne Catalyst Approach: A Cost-Effective and Robust Solution to Address the Requirements of BS-VI for Diesel Vehicles," SAE Technical Paper 2017-26-0127 (2017)

BAI, S.; TANG, J.; WANG, G.; LI, G. Soot loading estimation model and passive regeneration characteristics of DPF system for heavy-duty engine. Applied Thermal Engineering, 100, p. 1292-1298, 2016/05/05/ 2016.

CHOI, B.; LIU, B.; JEONG, J.-W. Effects of hydrothermal aging on SiC-DPF with metal oxide ash and alkali metals. Journal of Industrial and Engineering Chemistry, 15, n. 5, p. 707-715, 2009/09/25/ 2009.

COOK, S. L.; RICHARDS, P. J. An approach towards risk assessment for the use of a synergistic metallic diesel particulate filter (DPF) regeneration additive. Atmospheric Environment, 36, n. 18, p. 2955-2964, 2002/06/01/ 2002.

LACARRIERE, A.; SEGUELONG, T.; HARLE, V.; FABRE, C. A Low Ash and Highly Stable Formulated Fuel Borne Catalyst with Injection System Deposit Prevention Properties, 2015, SAE International, DOI: https://doi.org/10.4271/2015-01-0896.

LACARRIERE, A.; SEGUELONG, T.; SPIVEY, D.; DAS, A. The Fuel-Borne Catalyst Approach: A Cost-Effective and Robust Solution to Address the Requirements of BS-VI for Diesel Vehicles, 2017, SAE International, DOI: https://doi.org/10.4271/2017-26-0127.

MENG, Z.; CHEN, C.; LI, J.; FANG, J. et al. Particle emission characteristics of DPF regeneration from DPF regeneration bench and diesel engine bench measurements. Fuel, 262, p. 116589, 2020/02/15/ 2020.
ROCHER, L.; SEGUELONG, T.; HARLE, V.; LALLEMAND, $M$. et al. New generation fuel borne catalyst for reliable DPF operation in globally diverse fuels. SAE Technical Paper. 2011. (0148-7191).

SHADDICK, G.; THOMAS, M. L.; MUDU, P.; RUGGERI, G. et al. Half the world's population are exposed to increasing air pollution. Climate and Atmospheric Science, 3, n. 1, p. 23, 2020/06/17 2020. 
